\title{
Temperature Evolution in Nanoscale Carbon-Based Memory Devices due to Local Joule Heating
}

\author{
T. A. Bachmann, Student Member, IEEE, A. M. Alexeev, W. W. Koelmans, F. Zipoli, A. K. Ott, \\ C. Dou, A. C. Ferrari, V. K. Nagareddy, Member, IEEE, M. F. Craciun, V. P. Jonnalagadda, A. Curioni, \\ A. Sebastian, Senior Member, IEEE, E. Eleftheriou, Fellow, IEEE, and C. D. Wright, Member, IEEE
}

\begin{abstract}
Tetrahedral amorphous (ta-C) carbon-based memory devices have recently gained traction due to their good scalability and promising properties like nanosecond switching speeds. However, cycling endurance is still a key challenge. In this paper, we present a model that takes local fluctuations in $\mathbf{s p}^{2}$ and $\mathrm{sp}^{3}$ content into account when describing the conductivity of ta-C memory devices. We present a detailed study of the conductivity of ta-C memory devices ranging from ohmic behaviour at low electric fields to dielectric breakdown. The study consists of pulsed switching experiments and device-scale simulations, which allows us for the first time to provide insights into the local temperature distribution at the onset of memory switching.
\end{abstract}

\section{INTRODUCTION}

$\mathbf{O}$ $\mathrm{NE}$ of the emerging candidates to bridge the gap between fast but volatile DRAM [1] and non-volatile but slow storage devices is tetrahedral amorphous carbon (ta-C) based memory [2]-[5]. It offers a very good scalability, data retention and sub-5 ns switching speeds [3], [4]. Amorphous carbon memory devices can be electrically and optically switched from the high resistance state (HRS) into the low resistance state (LRS) [6]. The electrical conduction in the LRS is thought to be through $\mathrm{sp}^{2}$ clusters that form a conductive filament [5], [6].

Joule heating is assumed to be a primary contributor to resistive switching in ta-C [6]. The conductivity varies locally on the nanometer scale due to randomly distributed $\mathrm{sp}^{3}$ and $\mathrm{sp}^{2}$ sites [7]-[11] and hence, large local differences in current densities and hence in Joule heating can be expected.

A key challenge for carbon-based memory is endurance that is the number of cycles devices can be switched between LRS and HRS [4].

Excessive Joule heating could lead to the formation of large spatially-extended conductive filaments that make reversible switching back to the HRS difficult to achieve [10]. High temperatures and high current densities could also degrade the

T. A. Bachmann, A. M. Alexeev, V. K. Nagareddy, M. F. Craciun, and C. D. Wright are with the College of Engineering, Mathematics and Physical Sciences, University of Exeter, Exeter EX4 4QF, United Kingdom. (e-mail: tb352@exeter.ac.uk)

W. W. Koelmans, F. Zipoli, V. P. Jonnalagadda, A. Curioni, A. Sebastian, and E. Eleftheriou are with IBM Research - Zurich, Säumerstrasse 4, 8803, Rüschlikon, Switzerland

A. K. Ott, C. Dou, and A. C. Ferrari are with the Cambridge Graphene Centre, University of Cambridge, Cambridge, CB3 0FA, United Kingdom

2017 IEEE. Personal use of this material is permitted. However, permission to use this material for any other other purposes must be obtained from the IEEE by sending a request to pubs-permissions@ieee.org. electrodes as the devices are switched back and forth multiple times.

Here, we investigate Joule heating and the resulting temperature distributions within ta-C memory devices at the onset of memory switching. This is essential to gain further insights into the switching mechanism and to address the key challenge of cycling endurance. The model we present accounts for both electric field and temperature dependence of the electrical conductivity in ta-C. We also consider local distributions of $\mathrm{sp}^{2}$ and $\mathrm{sp}^{3}$-rich clusters. The simulations are validated with experimental data.

\section{Memory Device And Characterisation}

The memory devices consist of Pt (W) bottom (top) electrodes and $\mathrm{a} \mathrm{SiO}_{2}$ layer that has cylindrical openings of $50 \mathrm{~nm}, 100 \mathrm{~nm}$ and $200 \mathrm{~nm}$ diameter. The opening contains a $5 \mathrm{~nm}$-thick layer of ta-C deposited by filtered cathodic vacuum arc on top of the Pt. The remainder of each opening is filled with $\mathrm{W}$, see Figure 1 . The $\mathrm{sp}^{3}$ content is determined to $\approx 50 \%$ using multi-wavelength Raman spectroscopy [12]. On-chip load resistors in series with the memory device limit the current flowing through the device during the SET (i.e. switching to LRS) process. The series resistors, $3 \mathrm{k} \Omega$ to $14 \mathrm{k} \Omega$, are fabricated (e-beam lithography) next to the ta-C device and have a very small footprint to reduce their parasitic capacitance and hence the capacitive current flowing through the device during the SET process.

The reverse (RESET) switching process from LRS to HRS is reported to be induced by a large temperature gradient

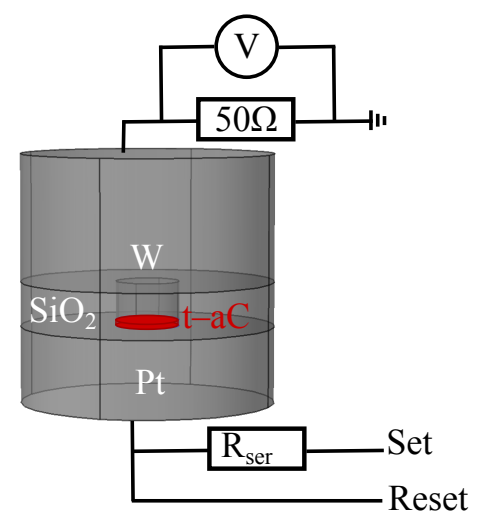

Figure 1. Schematic of the ta-C memory device with the electronic connections. SET and RESET pulses are applied to the bottom electrode. A load resistor limits the current during the SET process. 
[4], [13] that arises between the conductive filament and the insulating matrix once an electric pulse is applied. Hence, no load resistor is used during the RESET process. The read out of the current during the programming is done across a $50 \Omega$ resistor using an oscilloscope. The device resistance is read out using a Source Measurement Unit (SMU). A schematic of the memory device and the electrical circuit is shown in Fig. 1. More fabrication details and information on the electrical equipment used for the switching studies can be found in [4].

To study both the field dependence and the temperature dependence of the ta-C conductivity, we measure the lowfield conductivity of pristine devices (HRS) for temperatures from $85 \mathrm{~K}$ to $300 \mathrm{~K}$ using a cryogenic probing station (JANIS ST-500-2-UHT). The conductivity is plotted against $T^{-1 / 4}$ in Fig. 2 [14], [15]. The conductivity can be fitted by a straight

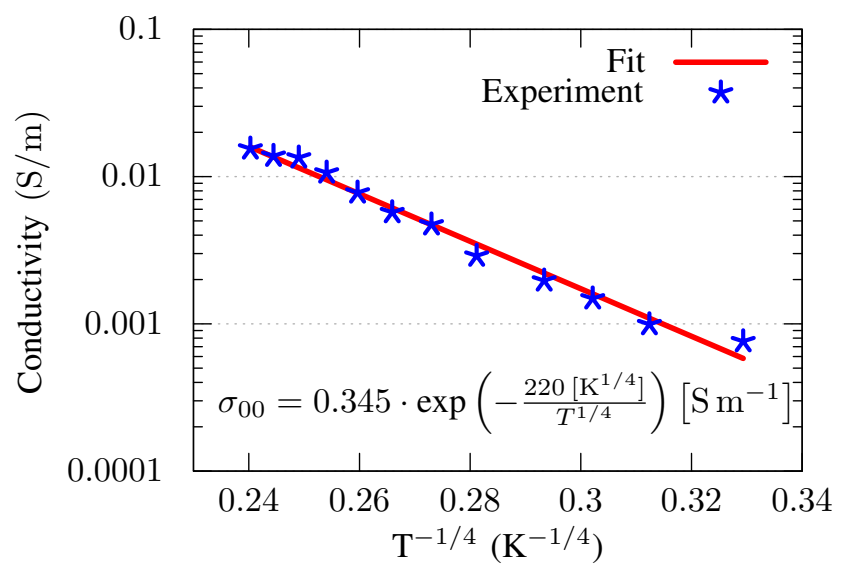

Figure 2. Conductivity of a carbon memory device measured at low voltages from $85 \mathrm{~K}$ to $300 \mathrm{~K}$.

line over the whole temperature range which indicates that the electrical transport is mainly governed by hopping between localised states [16]-[18].

For intermediate and high electrical fields we found agreement with a Poole-type conduction behaviour $(\log \sigma \sim E)$ for the field-dependent part of the conductivity (see section IV-B) [19], [20]. A Poole-type conduction behaviour is in agreement with reports of a high defect density in ta-C [21]. The transition from ohmic conduction to exponentially field-dependent conductivity can be described with a hyperbolic sine function as reported in [22], [23].

First, we look at experimental switching curves for voltage pulses of different durations. This allows us to examine the time response of the current on the applied voltage and, as a consequence, enables us to select a pulse long enough to exclude any "undesirable" transient effects prior to memory switching (e.g., due to thermal capacitances [24]). This, in turn, allows us to obtain a realistic temperature profile of the memory device at the onset of memory switching.

Therefore, we compare device I-V characteristics for a typical trapezoidal voltage with a $15 \mathrm{~ns}$ leading edge (LE) followed by a $45 \mathrm{~ns}$ plateau and a $15 \mathrm{~ns}$ trailing edge (TE) [4] with a quasi-static triangular pulse with $5 \mu \mathrm{s}$ LE and TE. The AC voltage outputs were supplied by an Agilent 81150A Arbitrary Waveform Generator and captured with a Tektronix

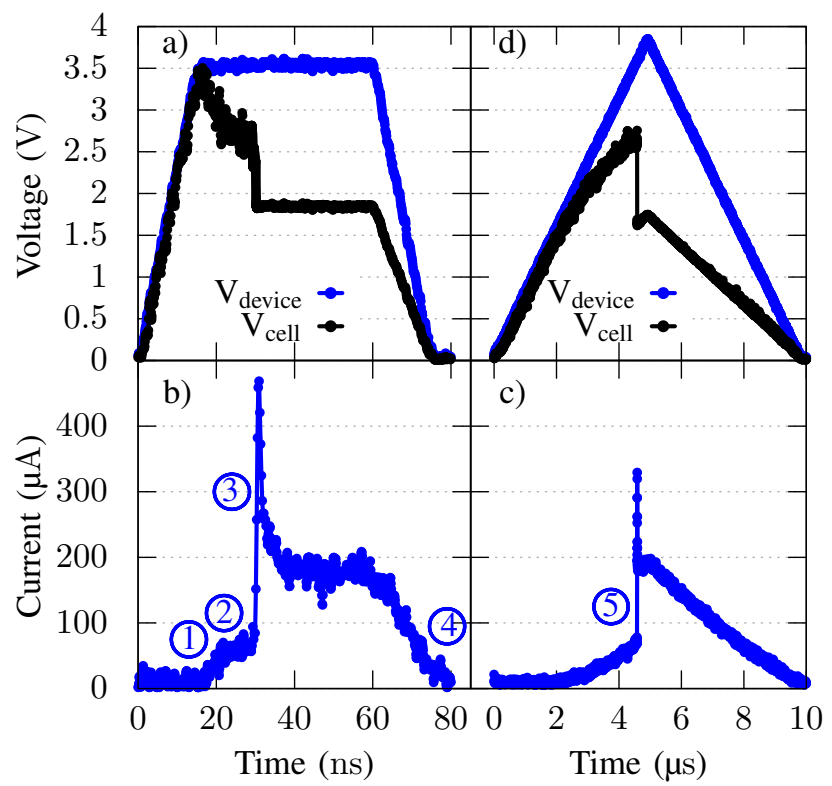

Figure 3. Applied voltage over devices (blue) and cells (black) together with the corresponding current response for a),b) fast $(80 \mathrm{~ns})$ and c),d) slow $(10 \mu \mathrm{s})$ pulses.

TDS3054B oscilloscope. The voltages applied across the device are plotted (blue) in Fig. 3 together with the voltage drop across the ta-C cell (black) and the corresponding currents. For noise reduction a $200 \mathrm{MHz}$ software filter is applied to the current and voltage signals during post-processing with the exception of the actual switching event.

The voltage pulse in Fig. 3a) reaches the plateau of the trapezoidal pulse after $15 \mathrm{~ns}$, during which little or no increase in current, see Fig. 3b), is noted (1). The current then begins to increase (2) and a dielectric breakdown sets in after $30 \mathrm{~ns}$ (3). From then on the current follows the voltage pulse (4). This indicates that the electric field alone, in the absence of sufficiently large currents, does not trigger memory switching.

No such time lag can be observed for the slow, quasistatic pulse. There, the current, see Fig. 3c), always follows the voltage pulse Fig. 3d) until dielectric breakdown occurs after $4.8 \mu \mathrm{s}$ (5) and the device is switched from its HRS into the LRS. Therefore, to validate the simulations we used a quasistatic pulse with $5 \mu$ s leading and trailing edge.

\section{MOdEL IMPLEMENTATION}

To obtain the temperature distribution in the device when a voltage is applied we use a finite element software package (COMSOL $®$ ) to solve the coupled heat and Laplace equations.

To reflect local $\mathrm{sp}^{2}$ variations in our model we randomly distribute different $\mathrm{sp}^{2}$-rich cluster concentrations within the simulation cells.

To ensure that a relatively small number of $\mathrm{sp}^{3}$-defects within a graphitic plane suffices to interrupt a conjugated $\pi$ network (see [8]) and to account for the $\mathrm{sp}^{2}$ bonded clusters that do not participate in the conduction, we set the threshold for $\mathrm{sp}^{2}$-like conduction to $92 \%$ within each simulation cell. The simulated initial conductivity for a ta-C cell with randomly distributed $\mathrm{sp}^{2}$ clusters is shown in Fig. 4. 


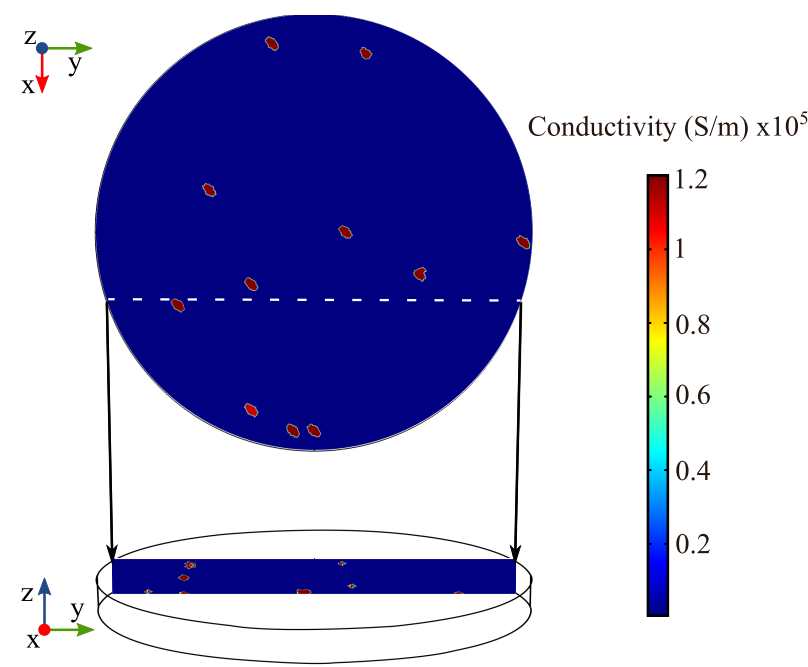

Figure 4. Initial conductivity for randomly distributed $\mathrm{sp}^{2}$-like conductive clusters (red); top shows conductivity in the x,y-plane (at $z=3.3 \mathrm{~nm}$ ), bottom shows conductivity in the $\mathrm{y}, \mathrm{z}$-cross-section (indicated by the dotted line).

An electric circuit was added to the electro-thermal model to account for a load resistor which, as seen in section II, is typically used to limit the current in the device after it has switched into the LRS.

The most important material properties used in the simulation are given in Table I.

Table I

MATERIAL PARAMETERS

\begin{tabular}{|c|c|c|}
\hline $\mathrm{sp}^{3}$-content $(\%)^{1}$ & & 50 \\
\hline $\operatorname{Beta}(\alpha, \beta)^{2}$ & & $2.65,2.65$ \\
\hline$\sigma_{\mathrm{sp}^{2}}\left(\mathrm{~S} \mathrm{~m}^{-1}\right)(\mathrm{cf} .[13])$ & & $1.2 \times 10^{5}$ \\
\hline$\sigma_{\mathrm{sp}^{3} \text {.ohmic }} @ 300 \mathrm{~K}\left(\mathrm{~S} \mathrm{~m}^{-1}\right)$ & & 0.0115 \\
\hline$\sigma_{00}\left(\mathrm{~S} \mathrm{~m}^{-1}\right)$ & & $0.345 \cdot \exp \left(-\frac{220\left[\mathrm{~K}^{1 / 4}\right]}{T^{1 / 4}}\right)$ \\
\hline$\sigma_{\mathrm{sp}^{3}}\left(\mathrm{~S} \mathrm{~m}^{-1}\right)$ & $\sigma_{00} \cdot \sinh$ & $\left(\frac{E}{9.5 \cdot 10^{9}\left[\frac{\mathrm{V}}{\mathrm{m}}\right]}\right)+\sigma_{\mathrm{sp}^{3}, \text { ohmic }}$ \\
\hline Threshold $\sigma_{\mathrm{sp}^{2}}(\%)$ & & 92 \\
\hline$\rho\left(\mathrm{kg} \mathrm{m}^{-3}\right)[25]$ & & $3460-1880 \times s p^{2}$ \\
\hline$\lambda\left(\mathrm{W} \mathrm{K}^{-1} \mathrm{~m}^{-1}\right)[26]$ & & $1.77 \cdot \rho-2.82$ \\
\hline$C_{p, a v g}\left(\mathrm{~J} \mathrm{~kg}^{-1} \mathrm{~K}^{-1}\right)^{3}$ & & 2050 \\
\hline
\end{tabular}

\footnotetext{
${ }^{1}$ Determined using multi-wavelength Raman spectroscopy

${ }^{2}$ Beta distribution with parameters $\alpha$ and $\beta$

${ }^{3}$ Average heat capacity of a memory cell computed from molecular dynamic simulations
}

\section{REsults \& Discussion}

\section{A. Isothermal Field-Dependent Conductivity}

To validate our model and to determine the field-dependent part of the conductivity we compare our simulation results with an experimentally measured device conductivity (Keithley $2636 \mathrm{~B}$ SMU). We use voltages high enough $(\approx 0.1 \mathrm{~V})$ to observe the exponential dependence of the conductivity on the applied voltage, but low $(\approx 1.1 \mathrm{~V})$ enough to keep the Joule

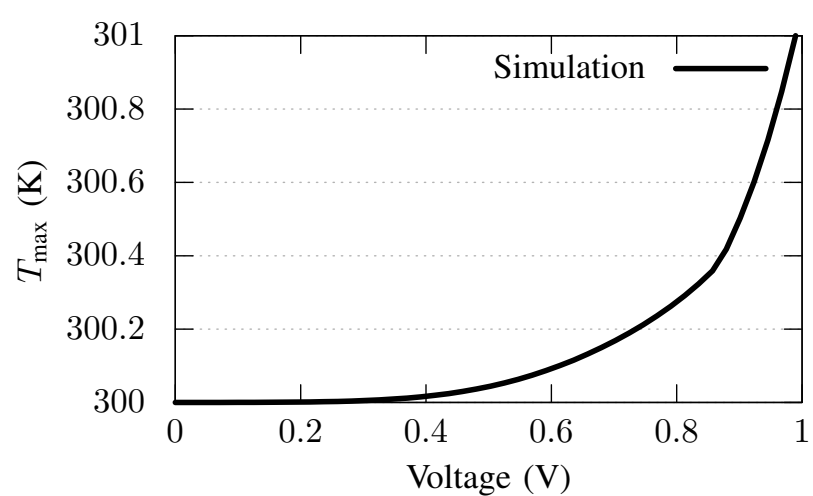

Figure 5. Local temperature evolution as function of the applied voltage.

heating below $1 \mathrm{~K} .{ }^{4}$ The maximum temperature within the cell is plotted as function of the applied voltage in Fig. 5. The simulated conductivity is compared with experimental data in Fig. 6. The simulation (red) describes the isothermal part of the conductivity (blue) very well up to electric fields of around $2 \times 10^{8} \mathrm{~V} \mathrm{~m}^{-1}$.

\section{B. Joule Heating Effects at Dielectric Breakdown}

To investigate Joule heating effects at the onset of dielectric breakdown we apply the triangular switching pulse shown in Fig. 3d). For both, experiment and simulation a $13.3 \mathrm{k} \Omega$ load resistor is used. The oscilloscope is programmed to

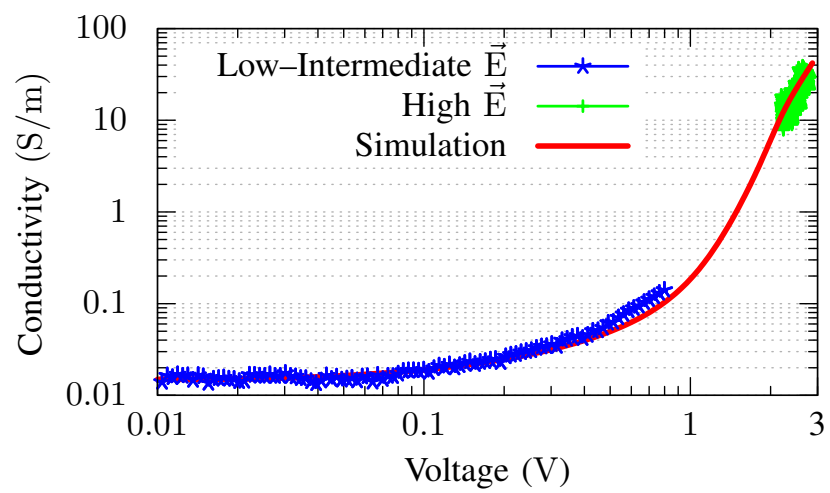

Figure 6. Measured and simulated conductivity of a carbon memory device as a function of the absolute value of the applied voltage.

ensure to capture the currents present at dielectric breakdown and as a consequence is insensitive to low currents at low voltages. Therefore, we only present here experimental highfield data for voltages from $|V|=2.2 \mathrm{~V}$ until the onset of the dielectric breakdown (i.e. $|V|=2.7 \mathrm{~V}$ ). The whole current vs. time response is depicted in Fig. 3c). The experimental and simulated conductivities are shown in Fig. 6.

The simulation describes the experimentally determined conductivity (blue, green) for all electric field ranges up to the point where dielectric breakdown $(|V|=2.7 \mathrm{~V})$ occurs very well. The current flowing through the device at the onset of the dielectric breakdown is $|I|=40 \mu \mathrm{A}$.

\footnotetext{
${ }^{4}$ The temperature increase was verified a posteriori with simulation results.
} 


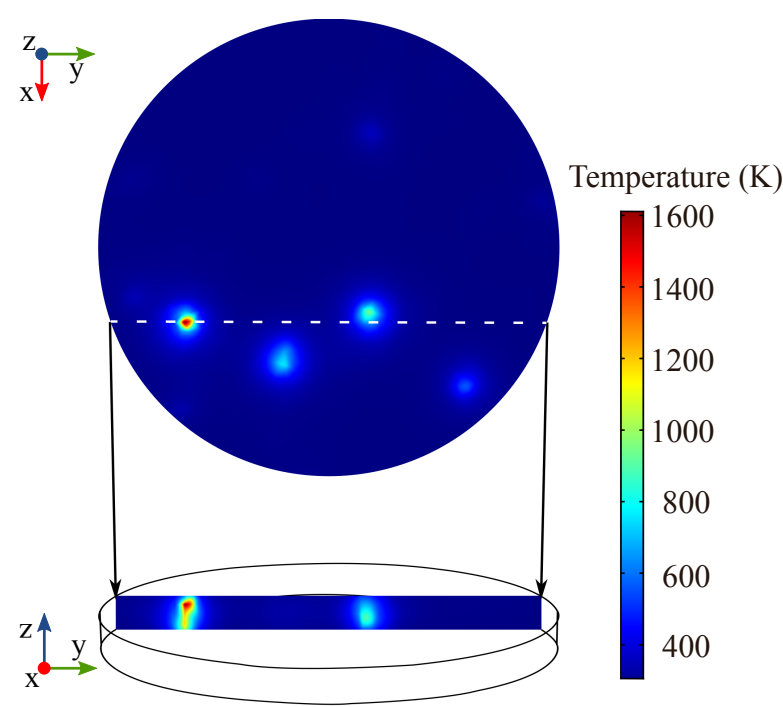

Figure 7. Temperature distribution at dielectric breakdown; top shows distribution in the $\mathrm{x}, \mathrm{y}$-plane (at $z=3.3 \mathrm{~nm}$ ), bottom shows distribution in the $y, z$-cross-section (indicated by the dotted line); applied voltage is $-3.2 \mathrm{~V}$.

The very good agreement between simulated and experimentally determined conductivity allows us for the first time to confidently investigate the local temperature distribution on the device-scale using a realistic switching pulse (see Fig. $3 \mathrm{~d}$ ). The temperature distribution within the memory cell is thus presented in Fig. 7.

The highest temperatures are obtained at $z=3.3 \mathrm{~nm}$, close to the mid-plane of the $5 \mathrm{~nm}$ thick ta-C layer. The electrodes remain close to room temperature, $311 \mathrm{~K}$ at the $\mathrm{Pt} / / \mathrm{ta}-\mathrm{C}$ interface and $337 \mathrm{~K}$ at the $\mathrm{W} / /$ ta-C interface which is expected as the metal electrodes act as heat sinks [4]. The observed high temperatures close to the mid-plane of up to $1615 \mathrm{~K}$ are in agreement with reports from molecular dynamic (MD) simulations [4]. The obtained hot spots are likely to indicate the origin where filament formation(s) after dielectric breakdown take(s) place.

These findings emphasise the relevance of taking local variations in conductivity into account and underline the importance of numerical modelling to obtain a detailed temperature profile of the memory cell which is not accessible otherwise. To illustrate this, a comparison between the average temperature in the memory cell and the maximum temperature is plotted in Fig. 8 as a function of the absolute value of the voltage drop across the memory cell.

The average temperature of the cell remains at moderate temperatures and reaches a maximum value of $320 \mathrm{~K}$, which agrees well with values reported in [27].

This finding is important as it emphasises that localised Joule-heating does not significantly affect the average temperature within the memory cell as long as the size of the hot spot is negligible in comparison to the lateral dimensions. Also, the highly localised hot spots prior to a filament formation provide evidence that memory switching in carbon-based devices is a temperature activated process which is in agreement with reports from MD simulations [4], [28], [29]. Note that an increase of the $\mathrm{sp}^{2}$ content would lead to a reduced distance

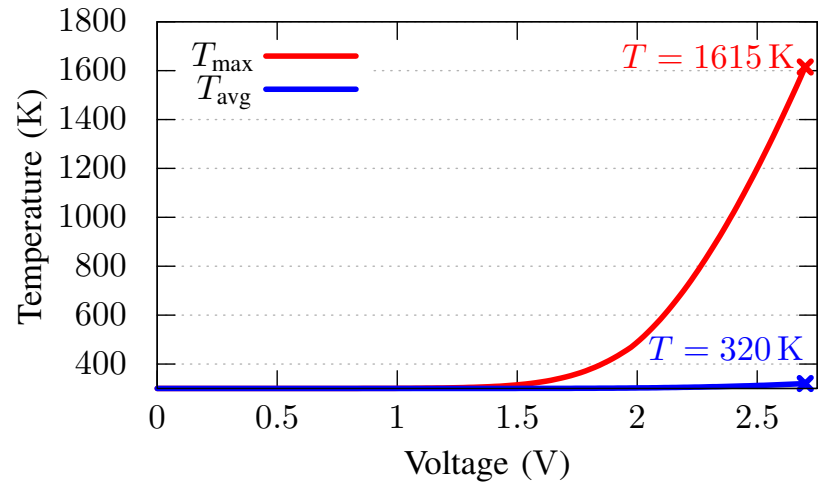

Figure 8. The maximum temperature inside the memory cell is plotted together with the average temperature as a function of the absolute value of the voltage across the memory cell.

between the $\mathrm{sp}^{2}$ conducting clusters in the $\mathrm{sp}^{3}$ matrix. This in turn would result in larger currents and higher temperatures due to Joule heating being more effective. Once the lateral dimensions of the device approach the filament dimensions, it is suggested that the whole device can be re-amorphized using a standard reset pulse, which would increase the cycling endurance significantly [3].

\section{CONCLUSiON}

We have successfully developed a simulation model that uses randomly distributed $\mathrm{sp}^{2}$ and $\mathrm{sp}^{3}$-rich clusters to constitute the material composition of ta-C memory devices. We have shown that by carefully taking the temperature and fielddependence of the $\mathrm{sp}^{3}$ conductive clusters into account we are able to reproduce an experimentally obtained conductivity ranging over $\approx 4$ orders of magnitude, all the way from ohmic conduction until dielectric breakdown. The simulation takes the overall $\mathrm{sp}^{3}$ content into account and therefore reflects that an increasing $\mathrm{sp}^{3}$ content will increase the resistivity and in turn a higher voltage will be required to produce a sufficiently high current density to induce dielectric breakdown.

As a result we have shown that Joule heating causes locally very high temperatures that, in turn, trigger a temperature activated process leading to material modifications. This temperature dependence emphasises the importance of adequate current control during the SET operation, so that sufficient current passes through the device for memory switching to occur and, at the same time, the current is limited to improve the cycling endurance. For further endurance improvements our findings suggest to decrease the gap between the electrodes, which increases the temperature gradient within the device during reverse switching. Future work aims to describe the full cycling dynamics including dielectric breakdown and reversible switching by implementing a cluster re-organisation that is based on a rate equation approach.

\section{ACKNOWLEDGMENT}

The authors thank all collaborators and colleagues involved in this project, in particular from IBM Zurich: M. Le Gallo and U. Egger for their help with the electrical setup, and 
C. P. Rossell for fruitful discussions. We thank M. M. Aziz from the University of Exeter for fruitful discussions. This work was funded by the EU research \& innovation project CareRAMM, no. 309980.

\section{REFERENCES}

[1] J. A. Mandelman, R. H. Dennard, G. B. Bronner, J. K. DeBrosse, R. Divakaruni, Y. Li, and C. J. Radens, "Challenges and future directions for the scaling of dynamic random-access memory (DRAM)," IBM Journal of Research and Development, vol. 46, no. 2.3, pp. 187-212, 2002.

[2] C. A. Santini, A. Sebastian, C. Marchiori, V. P. Jonnalagadda, L. Dellmann, W. W. Koelmans, M. D. Rossell, C. P. Rossel, and E. Eleftheriou, "Oxygenated amorphous carbon for resistive memory applications," Nature Communications, vol. 6, Oct. 2015.

[3] F. Kreupl, R. Bruchhaus, P. Majewski, J. B. Philipp, R. Symanczyk, T. Happ, C. Arndt, M. Vogt, R. Zimmermann, A. Buerke, A. P. Graham, and M. Kund, "Carbon-based resistive memory," 2008 IEEE International Electron Devices Meeting, pp. 1-4, Dec. 2008.

[4] W. W. Koelmans, T. Bachmann, F. Zipoli, A. K. Ott, C. Dou, A. C. Ferrari, O. Cojocaru-Mirédin, S. Zhang, M. Wuttig, V. K. Nagareddy, C. D. Wright, V. P. Jonnalagadda, A. Curioni, A. Sebastian, and E. Eleftheriou, "Carbon-based resistive memories," in International Memory Workshop, 2016.

[5] D. Fu, D. Xie, T. Feng, C. Zhang, J. Niu, H. Qian, and L. Liu, "Unipolar Resistive Switching Properties of Diamondlike Carbon-Based RRAM Devices," IEEE Electron Device Letters, vol. 32, no. 6, pp. 803-805, Jun. 2011.

[6] A. Sebastian, A. Pauza, C. Rossel, R. M. Shelby, A. F. Rodríguez, H. Pozidis, and E. Eleftheriou, "Resistance switching at the nanometre scale in amorphous carbon," New Journal of Physics, vol. 13, no. 1, p. 013020, Jan. 2011.

[7] A. C. Ferrari, B. Kleinsorge, N. A. Morrison, A. Hart, V. Stolojan, and J. Robertson, "Stress reduction and bond stability during thermal annealing of tetrahedral amorphous carbon," Journal of Applied Physics, vol. 85 , no. 10, p. 7191, 1999.

[8] K. Takai, M. Oga, H. Sato, T. Enoki, Y. Ohki, A. Taomoto, K. Suenaga, and S. Iijima, "Structure and electronic properties of a nongraphitic disordered carbon system and its heat-treatment effects," Physical Review $B$, vol. 67 , no. 21 , pp. $214202-11$, Jun. 2003.

[9] X. Chen, J. P. Sullivan, T. A. Friedmann, and J. M. Gibson, "Fluctuation microscopy studies of medium-range ordering in amorphous diamond-like carbon films," Applied Physics Letters, vol. 84, no. 1, pp. 2823-2825, Apr. 2004.

[10] J. D. Carey and S. Silva, "Disorder, clustering, and localization effects in amorphous carbon," Physical Review B, vol. 70, no. 23, Dec. 2004.

[11] Y. Miyajima, J. M. Shannon, S. J. Henley, V. Stolojan, D. C. Cox, and S. R. P. Silva, "Electrical conduction mechanism in laser deposited amorphous carbon," Thin Solid Films, vol. 516, no. 2-4, pp. 257-261, Dec. 2007.

[12] A. C. Ferrari and J. Robertson, "Interpretation of Raman spectra of disordered and amorphous carbon," Physical Review B, vol. 61, no. 20, pp. 14095-14 107, 2000.

[13] S. Qin, J. Zhang, D. Fu, D. Xie, Y. Wang, H. Qian, L. Liu, and Z. Yu, "A physics/circuit-based switching model for carbon-based resistive memory with $\mathrm{sp} 2 / \mathrm{sp} 3$ cluster conversion," Nanoscale, vol. 4 , no. 20 pp. 6658-6663, 2012.

[14] N. F. Mott and E. A. Davis, Electronic processes in non crystalline materials; 2nd ed., ser. International series of monographs on physics. Oxford: Clarendon Press, 1979

[15] N. F. Mott, "Conduction in non-crystalline materials," Philosophical Magazine, vol. 19, no. 160, pp. 835-852, Apr. 1969.

[16] C. Godet, "Variable range hopping revisited: the case of an exponential distribution of localized states," Journal of Non-Crystalline Solids, vol. 299, pp. 333-338, Apr. 2002.

[17] - "Analytical derivation of the effective temperature for fielddependent hopping conductivity," Philosophical Magazine Letters, vol. 83, no. 11, pp. 691-698, Nov. 2003.

[18] S. Baranovski, Charge Transport in Disordered Solids with Applications in Electronics, ser. Baranovski/Charge Transport in Disordered Solids with Applications in Electronics, S. Baranovski, Ed. Chichester, UK: Wiley, Sep. 2006.

[19] H. H. Poole, "VIII. On the dielectric constant and electrical conductivity of mica in intense fields," The London, vol. 32, no. 187, pp. 112-129, Jul. 1916.
[20] R. M. Hill, "Poole-Frenkel conduction in amorphous solids," Philosophical Magazine, vol. 23, no. 181, pp. 59-86, Jan. 1971

[21] J. Robertson, "Diamond-like amorphous carbon," Materials Science \& Engineering R-Reports, vol. 37, no. 4-6, pp. 129-281, 2002.

[22] M. Ieda, "A Consideration of Poole-Frenkel Effect on Electric Conduction in Insulators," Journal of Applied Physics, vol. 42, no. 10, pp. 3737-3740, 1971.

[23] D. Ielmini and Y. Zhang, "Analytical model for subthreshold conduction and threshold switching in chalcogenide-based memory devices," Journal of Applied Physics, vol. 102, no. 5, p. 054517, 2007.

[24] M. Le Gallo, A. Athmanathan, D. Krebs, and A. Sebastian, "Evidence for thermally assisted threshold switching behavior in nanoscale phasechange memory cells," Journal of Applied Physics, vol. 119, no. 2, 2016.

[25] S. R. P. Silva, Properties of Amorphous Carbon. IET, 2003.

[26] M. Shamsa, W. L. Liu, A. A. Balandin, C. Casiraghi, W. I. Milne, and A. C. Ferrari, "Thermal conductivity of diamond-like carbon films," Applied Physics Letters, vol. 89, no. 16, pp. 161921-161921-3, Oct. 2006.

[27] L. Dellmann, A. Sebastian, P. Jonnalagadda, C. A. Santini, W. W. Koelmans, C. Rossel, and E. Eleftheriou, "Nonvolatile resistive memory devices based on hydrogenated amorphous carbon." ESSDERC, pp. 268-271, 2013.

[28] G. N. Shumkin, F. Zipoli, A. M. Popov, and A. Curioni, "Multiscale Quantum Simulation of Resistance Switching in Amorphous Carbon," Procedia Computer Science, vol. 9, pp. 641-650, 2012.

[29] Y. H. Y. He, J. Z. J. Zhang, X. G. X. Guan, L. Z. L. Zhao, Y. W. Y. Wang, H. Q. H. Qian, and Z. Y. Z. Yu, "Molecular Dynamics Study of the Switching Mechanism of Carbon-Based Resistive Memory," IEEE Transactions on Electron Devices, vol. 57, no. 12, pp. 3434-3441, Dec. 2010.

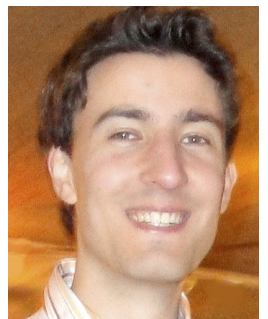

T. A. Bachmann (StM'17) received the B.Sc. and M.Sc. degrees in material science from ETH Zurich, Zurich, Switzerland, in 2011, and 2013, respectively. $\mathrm{He}$ is currently pursuing a Ph.D. degree with the School of Engineering, Mathematics and Physical Sciences, University of Exeter, Exeter, U.K.

From May 2015 to December 2015 he was a visitor in the Cloud and Computing Infrastructure Department at IBM Research Zurich, Zurich, Switzerland. His current research interests involve finite element simulations of electronic devices, electrical and surface characterisation of memory devices, in particular for storage applications.

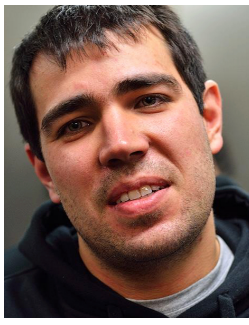

A. M. Alexeev received B.S. and M.S. in semiconductors engineering from the Saint-Petersburg State Polytechnical University, Russian Federation, in 2008, and the PhD degree in solid-state physics from the University of Exeter, Exeter, UK, in 2013.

Since 2013 he has been a Research Fellow with the Nano-Engineering Science Technology research group at the University of Exeter, UK. His research interests focus on developing novel electronic and optical devices based on carbon and phase-change materials. 


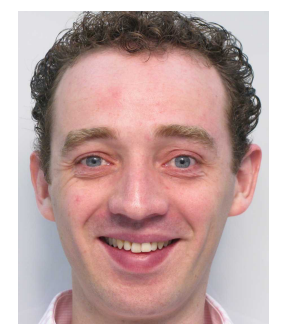

Wabe Koelmans received the M. Sc. and Ph. D. degrees in Electrical Engineering from the University of Twente, the Netherlands in 2006 and 2011, respectively. Since 2012, he is a scientist at IBM Research - Zurich in Rüschlikon, Switzerland.

His research interests are related to electronics, physics and mechanics on the micro- and nanometer scale. His research topics include emerging memory, brain-inspired computing, (scanning) probe technologies and sensors \& actuators as well as various other aspects of nanoscale science and engineering.

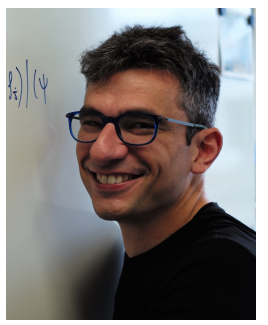

Federico Zipoli graduated in Materials Science at the University of Milano-Bicocca, Milan, Italy, in 2003, and obtained his Ph.D in Nanostructures and Nanotechnology in 2006, also in Milan. He is an expert in molecular-dynamics simulations, in firstprinciples calculations of electronic and structural properties of condensed systems, and in the study of chemical reactions and diffusion processes at surfaces. During his postdoc in the Department of Chemistry at Princeton University he studied via first-principles molecular-dynamics simulation the electrocatalytic hydrogen production from water by a biomimetic catalyst functionalizing a pyrite electrode. Since 2010 he is a Research Staff Member at IBM Research - Zurich in Rüschlikon, Switzerland. One of his research topic is modeling of phase-change and carbon-based materials for storage applications.

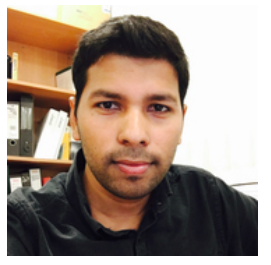

V. Karthik Nagareddy (M'16) received the M.Sc. degree in Nanoelectronics from the University of Manchester, UK in 2009 and the Ph.D. degree in electrical and electronic engineering from Newcastle University, UK in 2014. He is currently a Research Fellow at the University of Exeter, UK. His research interests include fabrication and characterisation of two-dimensional materials such as graphene, graphene oxide, $\mathrm{h}-\mathrm{BN}, \mathrm{MoS}_{2}, \mathrm{MoTe}_{2}$ and $\mathrm{WTe}_{2}$ for applications in electronics and optoelectronics.

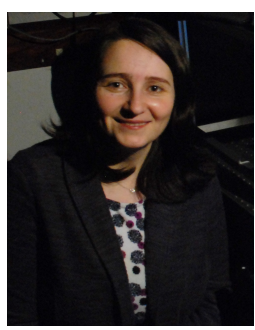

M. F. Craciun received the MSc in Physics from Joseph Fourier University, Grenoble, France and MSc in Applied Physics from the University of Bucharest Romania in 2001 and the MSc in Materials Engineering from the Catholic University Leuven, Belgium in 2004. She completed her PhD in Physics at Kavli Institute of Nanoscience, Delft University of Technology, The Netherlands in 2006. She was subsequently post doctoral researcher at The University of Twente, The Netherlands (2006/07) and at The University of Tokyo, Japan (2007/09) where she held a prestigious Fellowship of the Japanese Society for the Promotion of Science. She joined the University of Exeter in January 2010 as research fellow and took up the position of Associate Professor in Nanoscience in December 2014. The work of Prof Craciun spans from fundamental research in organic electronics and quantum electronics to applied research in electronic and optoelectronic materials and devices. Her current research focusses on two-dimensional materials for emerging technologies such as electronic textiles, smart windows and next generation of highly efficient optoelectronic devices. In 2014 she was awarded an early career fellowship from the U.K. Engineering and Physical Sciences Research Council and was investigator on more than 30 European Union- and U.K.-funded research projects.

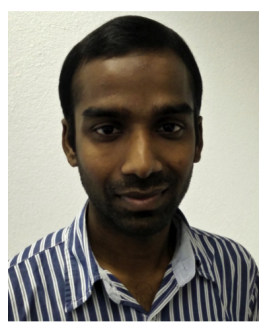

V. P. Jonnalagadda received the B.E. degree in electrical and electronics engineering from University of Madras, Chennai, India in 2004 and the M.SC. degree in Microsystems Engineering from Hochschule Furtwangen University, Furtwangen, Germany in 2007. From 2007 to 2010, he was a Research Assistant with the Institute of Applied research at Hochschule Furtwangen University, Furtwangen, Germany. Since 2011 he has been a process engineer with the Cloud and Computing Infrastructure Department at IBM Research - Zurich, Rüschlikon, Switzerland. His research interests focus on micro- and nanofabrication of non-volatile memory devices based on resistive and phase-change materials.

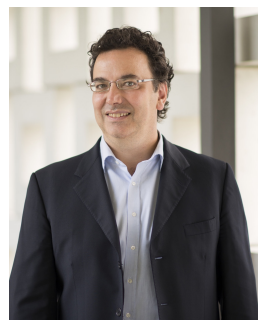

Alessandro Curioni received his diploma in Theoretical Chemistry and his $\mathrm{PhD}$ in Computational Materials Science from the Scuola Normale Superiore, Pisa, Italy. He joined IBM Research - Zurich in 1998 as Research Staff Member in the Computational Biochemistry and Materials Science Group. Since then he has developed and applied advanced computational methods to the study of materials issues related to IBM's core and exploratory technologies and to the investigation of important molecular processes in life sciences. In 2014, Dr. Curioni was promoted to the rank of IBM Fellow. In April 2015, he was named Vice President, Europe and Director of the IBM Research - Zurich Lab in April 2015. Since November 2015 he is leading the research activities for the newly formed Watson IoT unit.

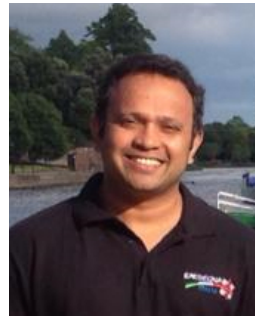

Abu Sebastian (SM'11) received a B. E. (Hons.) degree in Electrical and Electronics Engineering from BITS Pilani, India, in 1998 and M.S. and $\mathrm{Ph} . \mathrm{D}$. degrees in Electrical Engineering from Iowa State University in 1999 and 2004, respectively. Since 2006, he is a Research Staff Member at IBM Research - Zurich in Rüschlikon, Switzerland. His research has spanned several topics broadly related to dynamics and control at the nanometer scale. Research interests include emerging memory, storage and computing technologies as well as various aspects of nanoscale science and engineering. Dr. Sebastian is a co-recipient of the 2009 IEEE Control Systems Technology Award and the 2009 IEEE Transactions on Control Systems Technology Outstanding Paper Award. In 2013 he received the IFAC Mechatronic Systems Young Researcher Award for his contributions to the field of mico-/nanoscale mechatronic systems. In 2015 he was awarded the European Research Council (ERC) consolidator grant and in 2016 he was named an IBM master inventor.

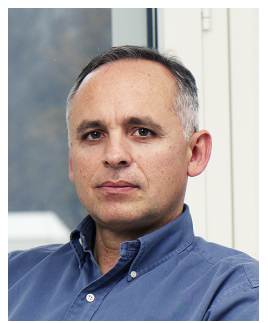

Evangelos Eleftheriou [S'81-M'86-SM'00-F'02] received his Ph.D. degree in Electrical Engineering from Carleton University, Ottawa, Canada in 1985.

He joined the IBM Research - Zurich laboratory in Rüschlikon, Switzerland, in 1986. Since 1998, he has held various management positions and currently heads the Cloud and Computing Infrastructure department of IBM Research - Zurich. In 2002, he became a Fellow of the IEEE. He was co-recipient of the 2003 IEEE Communications Society Leonard G. Abraham Prize Paper Award. He was also co-recipient of the 2005 Technology Award of the Eduard Rhein Foundation. In 2005, he was appointed an IBM Fellow and inducted into the IBM Academy of Technology. In 2009, he was co-recipient of the IEEE CSS Control Systems Technology Award and of the IEEE Transactions on Control Systems Technology Outstanding Paper Award. In 2016, he was elected Honoris Causa Professor at the University of Patras, Greece. 


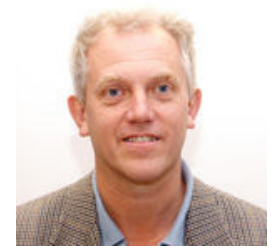

C. David Wright (M'96) received a B.Sc. degree in Physics in 1978 from Imperial College of Science and Technology, London and a Ph.D. in 'Perpendicular Magnetic Recording' from Manchester in 1985

He took up the Chair in Electronic and Computer Engineering at Exeter in 1999. His main research interests lie in the development of resistive-switching based materials and their use in a wide range of applications, from memories to computing to novel optoelectrics. He was the leader (co-ordinator) of the EU-FP7 project CareRAMM (Carbon resistive switching random access memory materials) that brought together partners from IBM Zurich, RWTH-Aachen, University of Cambridge, ISSP-Sofia and the University of Exeter, and under the auspices of which the work reported in this paper was carried out. He is Head of the Nano-Engineering, Science and Technology Group at Exeter, and is leader of the University's strategic research theme of 'Functional Materials'. 